\title{
Building an innovative Medical School in Portugal: the experience of the University of Minho
}

\author{
Nuno Sousa, Joana Palha, Jorge Pedrosa, Cecília Leão
}

Summary. 'Hallelujah!', proclaimed in October 8, 2001, 27 years after the first proposal delivered to the Portuguese government, the project's founder, Professor Joaquim Pinto Machado, at the beginning of educational activities at the School of Medicine (formerly School of Health Sciences), University of Minho (SM-UMinho), Braga, Portugal. This manuscript reviews the strategy implemented and the outcomes 15 years after welcoming the first medical students.

Key words. Educational process. Medical school. Medicine.

\section{Construyendo una facultad de medicina innovadora en Portugal: la experiencia de la Universidad de Minho}

Resumen. 'iAleluya!'. Así, 27 años después de la primera propuesta presentada al gobierno portugués, el fundador del proyecto, el Profesor Joaquim Pinto Machado, proclamaba el 8 de octubre de 2001 el inicio de las actividades educativas de la nueva Facultad de Medicina (anteriormente Escuela de Ciencias de la Salud) de la Universidad de Minho (SM-UMinho), Braga, Portugal. Este artículo revisa la estrategia utilizada para la implementación de la nueva facultad y los resultados 15 años después de dar la bienvenida a los primeros estudiantes de medicina.

Palabras clave. Facultad de medicina. Medicina. Proceso educacional.

\section{Introduction}

It is important to highlight that the opening of the SM-UMinho was associated with a clear purpose: to innovate. Filling in this aim and taking advantage of the unique opportunity to build from scratch, the SM-UMinho has an innovative pedagogical proposal that creates high quality educational and research processes.

\section{Mission and strategy}

The first step in constructing an educational building is the definition of its mission. Although it seems obvious, it is rather challenging to have a well-defined and visible mission for all the protagonists. The SM-UMinho clearly established as its mission 'to improve the quality of healthcare through education and research'.

To achieve this mission, the SM-UMinho defined a strong strategy based on three main vectors:

- Integration at the thematic faculty levels.

- Learning focused on evidence and on the clinical relevance that anticipates the needs of future medical practice.
- Engagement of every individual in the construction of his/her academic curriculum.

The study plan of the SM-UMinho is designed on the perspective of 'what should students learn and how can they learn best', which diverges critically from the strategy of 'what must we teach'. This change of standpoint is based on the founding principles of the new trends in medical education and encourages the application of active learning methodologies that promote the acquisition of cognitive skills and professionalism. In fact, herein lies the essence of critical operations in medical education (Fig. 1a) which, when associated with rigorous evaluation processes (Fig. 1b), allow the definition (and re-definition) of suitable educational objectives, ensuring a correct and effective alignment with the mission of the institution. Concomitantly, and of notice, it also ensures the flexibility to adjust and anticipate new needs. In this line and more recently, the thinking in medical education has given a greater focus to a system-based, competencybased educational model, where the health needs/ systems are equated to drive competencies outcomes, which are adequately assessed, to determine the curriculum.
ICVS/3B's, PT Government Associate Laboratory. Life and Health Sciences Research Institute (ICVS); School of Medicine; University of Minho; Braga, Portugal.

Correspondencia:

Prof. Nuno Sousa. School of Medicine. University of Minho. CP 4710-057. Braga, Portugal.

E-mail:

njcsousa@ecsaude.uminho.pt

Recibido:

09.01.17.

Aceptado:

10.01.17.

Conflicto de intereses: No declarado.

Competing interests: None declared.

(c) 2017 FEM 
Figura 1. Schematic representation of the conceptual flow and interplay within a Medical School: curricular elements (a) and 'operations' (b) in medical education. a

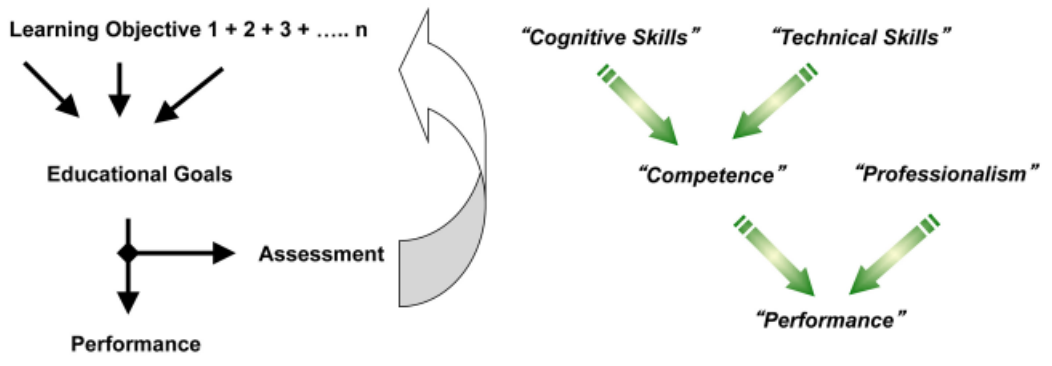

Table I. Adaptation of the data presented in Martins study [1], summarizing the 2006-2011 scores of the Portuguese candidates to the national residency selection exam.

\begin{tabular}{|c|c|c|c|c|c|c|c|}
\hline Medical School & $n$ & 1 & 2 & 3 & 4 & 5 & 6 \\
\hline Foreigners Medical Schools & 1107 & 48.85 & & & & & \\
\hline $\begin{array}{l}\text { Faculty of Health Sciences, } \\
\text { University of Beira Interior }\end{array}$ & 309 & & 63.84 & & & & \\
\hline Faculty of Medicine, University of Coimbra & 1597 & & 66.10 & 66.10 & & & \\
\hline Nova Medical School & 1400 & & & 66.26 & & & \\
\hline $\begin{array}{l}\text { Biomedical Institute Abel Salazar, } \\
\text { University of Porto }\end{array}$ & 972 & & & 67.30 & 67.30 & & \\
\hline Faculty of Medicine, University of Lisbon & 1835 & & & & 69.41 & 69.41 & \\
\hline Faculty of Medicine, University of Porto & 1472 & & & & & 69.95 & \\
\hline School of Medicine, University of Minho & 264 & & & & & & 72.33 \\
\hline Sig. & & 1.000 & 0.064 & 0.778 & 0.107 & 0.997 & 1.000 \\
\hline
\end{tabular}

Post-hoc analysis of average scores per Medical School. Groups within the same column do not present statistical differences amongst them. Means for groups in homogeneous subjects are displayed $(\alpha=0.05)$.

The implementation of such a strategic plan needs a stable leadership (but flexible), respected by the various stakeholders and with ability to run according to the principles set out in contexts that change frequently. As such, the leadership action should be based on a culture of freedom with strict scrutiny (also aligned with the underlying culture of assessment of the institution); ultimately, this allows the final goals and objectives to be achieved. The leadership of the SM-UMinho defined the following baselines to the implementation strategy:
- A study plan organized in learning objectives and focused in competence acquisition in the domains of knowledge, clinical skills, medical humanities, and professionalism.

- Multi-disciplinary functional curricular units.

- Multi-centric affiliations.

- Responsibilities that extend beyond graduation.

- Implementation of a quality monitoring system following the best international practices.

Fifteen years have passed, so here facts that illustrate the results achieved.

\section{A Medical School that endorses high quality educational processes}

The first fact regards the quality of the educational process. Surely the best way to assess the quality of such a process is through the results obtained by its participants. Thus, next are presented the overall results achieved by the students and graduates in various dimensions.

In Portugal, there is a national exam to rank the candidates for entering Medical Residency. The results from this exam were analyzed in an independent scientific article [1]; this study showed that in the years 2006 to 2011 the scores obtained by graduates of the SM-UMinho were, on average, significantly higher than those obtained by the candidates from other Portuguese or foreign medical schools (Table I). This result has been subsequently confirmed for the years 2012 to 2015 (data not shown). Despite the disputable quality of this exam, which is based on memory assessment of some chapters of the Harrison's Principles of Internal Medicine book, it is to note that the SM-UMinho graduates have since the beginning ranked first in that list. The relevance of such achievement should be considered also in light of the entry scores, also based in a national application process, and in which the students entering the SM-UMinho, from high school, do not rank first on average.

More relevant appear to be the results obtained in international assessments, including those of the International Foundation of Medicine (IFOM) Exam, an examination prepared by the National Board of Medical Examiners (NBME), or of the European Board examinations of Medical Assessors (EBMA) [2]. In both cases, students from SM-UMinho compared well with their colleagues in prestigious international medical schools. In the IFOM exam (Fig. 2), it is important to highlight that students from the fifth grade of the SM-UMinho were all 
above the cut score defined for that exam, which ensures that the adequate knowledge is obtained before students enter the sixth more professional year of their 6 year-long academic curriculum. Submitting students to these international standardized and validated exams adds useful information also on the syllabus of the training provided. In accordance, it is of notice that the contents/ competences acquired in the early stages of formation are stable in later outcomes (Fig. 2), providing evidence that consolidation of learning was achieved with the methodology applied on SM-UMinho.

Medical practice is not confined, however, to cognitive skills. Therefore, another important dimension of the quality of the educational processes is measured by the performance in clinical contexts. The SM-UMinho activities provide the tools/ environment for such competences to be achieved through simulation programs (for which the initial equipment support from the Fundação Calouste Gulbenkian was instrumental) and planned clinical clerkships. To monitor the real impact of such strategy on the clinical practice of our graduates, the SM-UMinho takes advantage from a longitudinal follow-up project that was launched in the beginning of the medical school with the intention to better characterize, monitor and understand the educational process. This longitudinal project has collected valuable information with this respect: in the opinion of their training clinical supervisors, the overwhelming majority of the SM-UMinho graduates $(97 \%)$ are in the top quartiles of performance when compared with students and graduates trained in other institutions (Table II). More relevant is to note that none of the SM-UM students is in the bottom quartile of performance (Table II).

As a whole, and combined with many other intermediate data properly collected, analyzed, advertised and scrutinized, the SM-UMinho has evidence to trust the quality of its educational process.

\section{A certified Medical School with prestige}

Regardless of the value given to the certifications, the truth is that these processes enable an internal and external assessment exercise, which builds confidence on what an institution is achieving. In this way, at the earliest opportunity, the SM-UMinho applied and was granted the certificate of excellence Aspire, an initiative promoted by the Association for Medical Education in Europe (AMEE) to recog-
Figura 2. Results obtained by the students of SM-UMinho in the 2008 International Foundations of Medicine (iFOM) exam. The blue line represents the cut-score established for the 2008 iFOM exam.
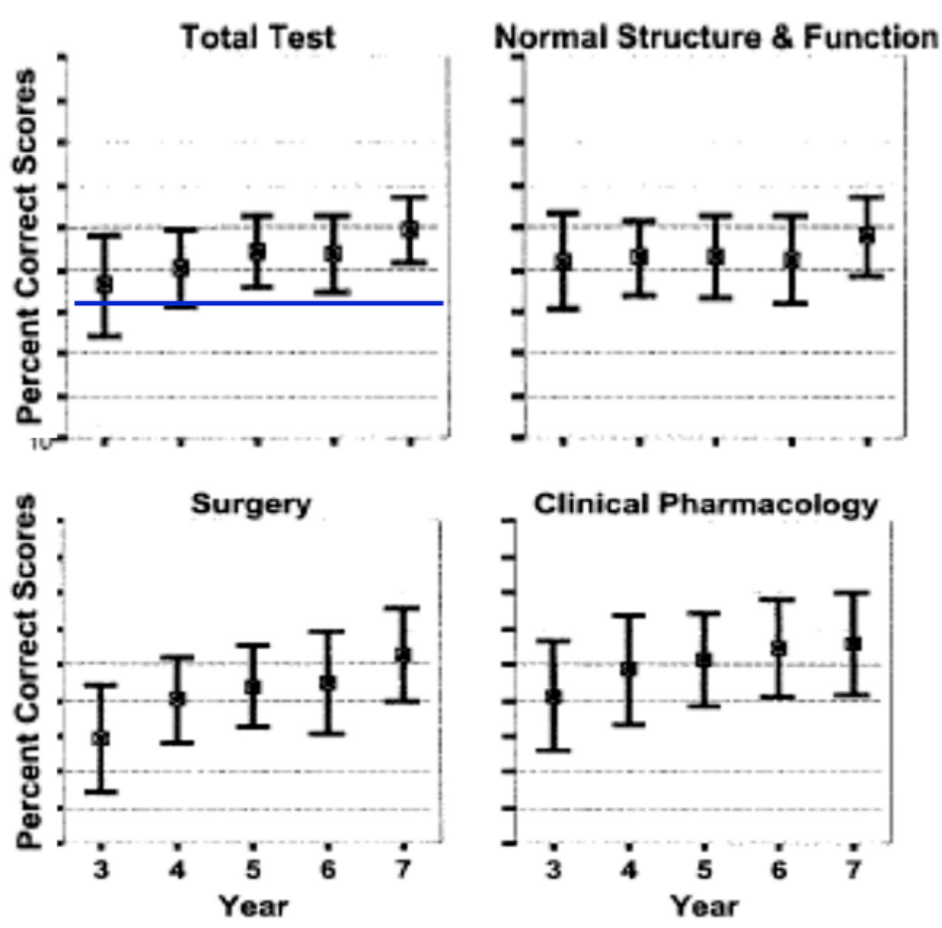

Table II. Distribution of performance by the SM-UMinho graduates, scored by their training clinical supervisors.

\begin{tabular}{lcc}
\hline & Knowledge & Professionalism \\
\hline Bottom quarter & 0 & 0 \\
\hline Lower middle quarter & $9(1.5 \%)$ & $9(3 \%)$ \\
\hline Upper middle quarter & $264(45 \%)$ & $106(39 \%)$ \\
\hline Top quarter & $265(45 \%)$ & $159(58 \%)$ \\
\hline Total & $538(91 \%)$ & $274(100 \%)$ \\
\hline
\end{tabular}

nize 'student engagement in the curriculum and in the school.' Only eight medical schools were distinguished with this certificate in the first round of applications, and the SM-UMinho the only European. This attests the present value of the project, but mostly increases the responsibility to continue to build a pedagogical community of excellence on this and other dimensions of the educational project. 
Figura 3. Schematic representation of the framework of the MD/PhD program at SM-UMinho.

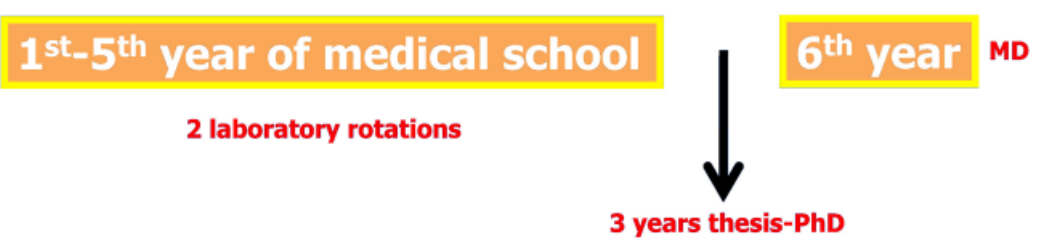

It must, at this point, be said that a volunteer open culture of assessment, internal and from external institutions and panels, constitutes the pillar of progress in the SM-UMinho medical training. The feedback obtained represents a reappraisal of decisions taken and an opportunity for continuous improvement, which is considered seriously by the School. Publicly reporting should also be regarded as a duty, and therefore the pedagogical results of the SM-UMinho have been published in various congresses, by its faculty, non-faculty members and students. Despite its short 16 years history, the SMUMinho has established numerous national and international partnerships. This sharing of results and experiences allowed the integration of SM-UMinho (or its members) in various national and international agencies, among which stands out the fact that the SM-UMinho is a founding member of the EBMA. The SM-UMinho is committed to contribute to the consolidation of the Medical Education community in Portugal.

The mission of the SM-UMinho is not confined to undergraduate medical training, but also to promote and offer continuous medical education, medical training and postgraduate opportunities. In this area, the SM-UMinho has invested in advanced training as a key approach not only to the biomedical and medical communities but also to contribute to generate value within the medical surrounding and in the creation of training actions for residents (with the creation of various formal activities recognized by Specialty Colleges and with the participation of a very high percentage of national residents), as well as in a recognized continuous medical education program based on more than 50 international courses and workshops/year and dedicated PhD programs in medicine and in health sciences, including a formal $\mathrm{MD} / \mathrm{PhD}$ program for a restricted number of medical students to develop a $\mathrm{PhD}$ before completion of the MD training.

\section{A Medical School with focus on research}

Another critical aspect in the development of SM-UMinho's educational project was the decision to install, within the Medical School, a research institute, the Life and Health Sciences Research Institute-ICVS, in contrast to the usual centrifugal strategy of the Portuguese scientific network. Thus, the ICVS, launched in 2003 and today a PortugueseGovernment Associated Laboratory, was created within a vision to fulfill the established mission.

The setting of the ICVS in the SM-UMinho building allows medical students proximity and constant contact with the research laboratories, where researchers with diverse backgrounds, including many of their professors, carry out research activities. In this way, and because students are given several opportunities to engage in research (the study plan has several optional units), the critical concept of a physician-scientist is intentionally fostered. To date, hundreds of projects have been developed by SM-UMinho students at the ICVS (and many more outside the ICVS), some of them giving rise to peer-reviewed publications and awards.

More importantly, this ecosystem allows the continuation of numerous research projects of students in extra-curricular activities, which resulted in a high percentage of students to continue their interest and passion for research after graduation. This aspect, of which the SM-UMinho project is very proud, has allowed the design of longer and more intense research projects, which later gave rise to formal post-graduation programs in health sciences and in medicine, among which the SMUMinho MD-PhD program stands out (Figure 3). This program, developed in partnership with the Columbia and Thomas Jefferson Medical Schools in the USA, is unique in Portugal and still one of the very few in Europe. It was recently distinguished as a program recognized and supported by the national founding agency Foundation for Science and Technology (FCT), after fundamental initial support of the Luso-American Foundation for Development (FLAD).

Therefore, the decision to launch a research institute within a medical school has to date been successful. It again constitutes an opportunity. In accordance, and lastly, the ICVS and the SM-UMinho established in Hospital de Braga a partnership with the José de Mello Saúde group and created a Clinical Academic Center (2CA-Braga), a unit dedicated to clinical research. Certainly, this step is aligned with the centrality of research in the context of a Medical School guided by international 
standards of quality. This partnership ensures the best conditions for the development of clinical research, a research area with an important handicap in Portugal. This joint effort, which started in 2012, is already bearing fruit, with the capture of a high percentage of the clinical trials ongoing in Portugal and with the development of a significant number of clinical research projects driven by researchers. These synergies allow the accomplishment of the mission of a research institute on biomedicine and clinical sciences: to promote research from the bench to the bedside and vice versa.

\section{The vision and the future}

The vision of the SM-UMinho leadership is that this ecosystem guarantees an innovative health cluster in the region (and perhaps scalable to the country).
In this cluster, it is possible, and should continue to be possible, to educate and train (and re-train) physicians, as well as other biomedical scientists and health professionals, creating the conditions for generating knowledge (and value) with clinical applicability and, ultimately, improving medical care of the populations. This must be and continues to be the challenge and commitment of the SM-UMinho daily activities and future planning.

\section{References}

1. Martins IP. Admission to residence training in Portugal: analysis of the National Exam results between 2006 and 2011 Acta Med Port 2013; 26: 569-77.

2. Winward ML, De Champlain AF, Grabovsky I, Scoles PV, Swanson DB, Holtzman KZ, et al. Gathering evidence of external validity for the Foundations of Medicine examination: a collaboration between the National Board of Medical Examiners and the University of Minho. Acad Med 2009; 84 (Suppl 10): S116-9. 